\title{
Historical evolution of spatial abilities
}

\author{
A. Ardila \\ Instituto Colombiano de Neuropsicología Bogotá, Colombia \\ Correspondence to: A. Ardila, Apartado Aéreo 17021, Bogotá, Colombia, South America
}

\begin{abstract}
Historical evolution and cross-cultural differences in spatial abilities are analyzed. Spatial abilities have been found to be significantly associated with the complexity of geographical conditions and survival demands. Although impaired spatial cognition is found in cases of, exclusively or predominantly, right hemisphere pathology, it is proposed that this asymmetry may depend on the degree of training in spatial abilities. It is further proposed that spatial cognition might have evolved in a parallel way with cultural evolution and environmental demands. Contemporary city humans might be using spatial abilities in some new, conceptual tasks that did not exist in prehistoric times: mathematics, reading, writing, mechanics, music, etc. Crosscultural analysis of spatial abilities in different human groups, normalization of neuropsychological testing instruments, and clinical observations of spatial ability disturbances in people with different cultural backgrounds and various spatial requirements, are required to construct a neuropsychological theory of brain organization of spatial cognition.
\end{abstract}

Keywords: Right hemisphere - Spatial agnosia - Spatial cognition - Spatial disturbances - Spatial orientation

\section{INTRODUCTION}

Contemporary city life, in which direct orientation in space has been replaced by the logical application of mathematical coordinates, not only represents a relatively recent cultural acquisition, but also, is only found in some contemporary human groups. For a very long time, education consisted of learning to orient ourselves in space, to recognize the pertinent signals to follow a prey, and to move in the surrounding spatial environment. This, of course, is still valid for contemporary people living in the Amazonian jungle, for Eskimos, for desert inhabitants, and many other world inhabitants. For thousands (and even millions) of years, human survival depended on the correct interpretation of spatial signals, memory of places, calculation of distances, and so forth, and the human brain must have become adapted precisely to handle this kind of spatial information (Ardila and Ostrosky, 1984).

The paleolithic age extended from about 2300000 years ago (first hominids using stone tools) until some 10000 years ago (Childe, 1936; Hours, 1982). About this time, agriculture appeared, and humans began to domesticate and raise animals. This produced a tremendous change in their way of life (the Neolithic Revolution). The paleolithic age represents in consequence the vast majority of humankind history (about $98 \%$ ).

It might be supposed that biological adaptation was accomplished to survive under those conditions existing during the paleolithic time. Recent human evolution corresponds more exactly to a cultural type of evolution, not necessarily requiring further biological changes, but requiring the development and use of those basic cognitive strategies already existing during the paleolithic time. Agriculture replaced fruit collection; domestication of animals replaced hunting; written language extended oral language; arithmetic extended finger counting; and the use of maps and logical spatial coordinates, the direct orientation in space (Vygotsky, 1962).

There is evidence that Australopithecus africanus (probably the direct ancestor of $\mathrm{Homo}$ ) lived on open savannas. Although their ancestors lived in forests, they moved to live in open fields (Lee and DeVore, 1968; Wilson, 1975). They strongly depended on animal food, especially small prey (tortoises, lizards, snakes, rabbits) and collecting fruit. Homo sapiens appeared in Europe about 50000 years ago (middle paleolithic), and it is supposed that they hunted, usually small but also big prey, used rudimentary stone tools (knives, axes), and lived in caverns or rudimentary shelters built with tree branches. They were nomadic and spent their time moving from one place to another looking for prey, fruit, and shelter. They created some hunting weapons like the arrow and the spear, and used fire (Washburn, 1978; Hours, 1982).

It might be supposed that spatial abilities were even more crucial for survival in prehistoric than in contemporary humans. Survival in current urban living conditions requires different cognitive abilities. We go around our cities using logical mathematical coordinates, reading a printed map, and without taking into consideration the sun's position in the sky, or avoiding potential predators. Adaptation to contemporary world conditions requires more verbally based abilities. If the question "What moon 
phase is it today?" (instead of "What date is it today?") were included in a mental state examination, the majority of city people would fail. By the same token, city people usually ignore the exact sunrise and sunset points (they usually respond "east and west"), but sunrise and sunset points change a little everyday, and it could have been an important piece of information for prehistoric human's survival, as it is for contemporary humans to know that “Today is 6 May 1990". Furthermore, our current educational system strongly emphasizes verbal, logical, and mathematical abilities, not spatial orientation abilities. Of course, these are currently the most useful abilities to survive in our contemporary world. Generally speaking, city people have limited opportunities to develop and use spatial orientation abilities.

\section{HOW DO WE GET ORIENTED IN SPACE?}

Cross-cultural differences in spatial orientation strategies under normal and pathological conditions could be illustrative in understanding how prehistorical humans could have used spatial information. Furthermore, it could shed some light on the potential spatial abilities that contemporary humans possess. Brain organization of spatial abilities under pathological conditions has been extensively studied in contemporary schooled Western (particularly European and North American) people. To the author's best knowledge, however, no clinical observations about disturbances in spatial abilities associated with brain pathology in other (non-Western) cultures have been reported.

Characteristics of spatial abilities in different cultures can be illustrative. Perceptual constancy (stability of perception despite changes in the actual characteristics of the stimuli) represents the most fundamental ability in the interpretation of the surrounding spatial environment (Ardila, 1980).

\section{Perceptual constancy}

Cross-cultural comparisons have in general demonstrated that perceptual constancy (size and shape constancy) is more accurate in people of low-schooled and non-Western societies than in literate and Westernized subjects (Pick and Pick, 1978). Beveridge (1940) demonstrated a greater constancy of shape and size perception among West African adults than among British adults. Myambo (1972) observed an almost perfect shape constancy in uneducated Malawi adults, whereas educated Africans and Europeans did not perform so accurately. Perceptual constancy may be expected to be present (and crucial for survival) not only in prehistoric humans, but also in people currently requiring a complex interpretation of the surrounding spatial environment.

\section{Reference systems}

People living in different environments develop different systems of spatial reference (rivers, mountains, sun position, streets, buildings, etc.). Geographic features affect the terms of local reference systems, and differences in reference systems may, in turn, be related to differences in perception of spatial orientation (Pick and Pick, 1978). The analysis of different reference systems can be illustrative.

Gladwin (1970) analyzed the system used by Puluwat sailors to navigate among clusters of islands in the western Pacific. He disclosed that many different features of the sea and sky comprise the information on which the system is based. Knowledge of the habits of local sea birds provides cues for one's location. The sailors learn to detect coral reef formation changes, which differ depending on the conditions of the weather, sea and sky. Ability to detect change in the "feel" of the boat moving through the waves on a particular course is a skill used to maintain a course. There is a complex reference system based on the position and patterns of stars in the night sky, and the rules for navigating between specific islands are described in terms of the star patterns and islands. Parallax information is also explicitly included in the system as descriptions of the way in which the islands "move" as the boat passes on one or the other side of them (Pick and Pick, 1978).

Amazonian Indians simultaneously use a variety of different types of information to move around in the jungle. They use small rivers, orientation and color of trees, soil characteristics, sun position, animal routes, olfactory cues, and many other signals. Vegetation is mildly different when closer to rivers, moss grows differently on trees according to the sun direction, and direction of river flow can differ. Additionally, when moving in the jungle, they break small bush branches, to recognize later that they have already crossed that particular point (and approximately how long ago). All these environmental signals are simultaneously interpreted for becoming oriented and moving around the jungle.

Evidently, members of different cultures dwelling in different spatial environments operate in terms of complex spatial reference systems, depending on their particular demands and geographic environments. Demands and geographic environment were quite different in paleolithic times from those experienced by contemporary city humans. Evidently, spatial orientation and reference systems used by prehistoric humans were closer to Puluwat sailors' or Amazonian Indians' reference systems, than to our contemporary orientation system in cities.

\section{Cultural differences in visuoperceptual abilities}

Cross-cultural differences in perceptual abilities have been extensively studied and can be particularly illustrative in understanding perceptual skills in prehistoric 
humans (Brislin, 1983; Laboratory of Comparative Human Cognition, 1983; Segall, 1986). Hudson (1960, 1962) studied depth perception using pictures that contained figures of an elephant, an antelope, and a man with a spear; the basic question referred to what the man was doing with the spear. There were four pictures differing with respect to the cues available for the interpretation of the picture. This set of pictures was used with different groups of people from Africa and Europe. It was observed that European children around 7-8 years have great difficulty perceiving the picture as three-dimensional. However, around 12 years, virtually all perceived the picture as three-dimensional. This was not the case with Bantu or Guinean children. Non-literate Bantu and European laborers responded to the picture as flat, not three-dimensional. They could not interpret three-dimensional figures represented on paper; this is also the case in general for illiterate people (Ardila et al., 1989). However, as mentioned above, illiterate African people do better than Western literate subjects in perceptual constancy tasks with real objects; but they do worse when the external space is represented on paper.

Berry $(1971,1979)$ proposed that hunting people with specific ecological demands usually present good visual discrimination and excellent spatial skills. For instance, the embedded figures test is better performed by cultural groups for whom hunting is important for survival. Berry emphasizes that ecological demands and cultural practices are significantly related to the development of perceptual and cognitive skills. A good example of a specific culturedependent cognitive skill was that reported by Gay and Cole (1967); when Kpelle farmers are contrasted with American working class, the former were considerably more accurate in estimating the amount of rice in several bowls of different sizes containing different amounts of rice. By the same token, any cattle farmer is able to calculate accurately the weight of a cow; or any dactilographist can easily and quickly distinguish two different fingerprints; or any neurologist can distinguish a Parkinsonian patient at one glance. Demands and training history are strongly associated with visuoperceptual abilities.

Spatial abilities differ among cultures and depend on the specific environmental demands. In neuropsychology, the perceptual ability disturbances of a very limited subsample of the human species-contemporary Western, and most often, urban and literate brain-damaged individuals-have been relatively well analyzed. Nevertheless, our understanding of the brain organization of spatial abilities, and their disturbances in cases of brain pathology, are necessarily not only partial but, doubtless, biased. Norms for perceptual performance in a sufficiently broad array of neuropsychological tests, and an extended analysis of perceptual disturbances in different cultural and environmental contexts, are required.

\section{ACQUIRED SPATIAL COGNITION DISORDERS}

Visuospatial impairments resulting from brain damage have been extensively analyzed in neuropsychology (e.g. Hécaen, 1962; De Renzi, 1982, 1985; Rosselli, 1986; Morrow and Ratcliff, 1988; Newcombe and Ratcliff, 1989). Different brain syndromes have been distinguished.

Spatial agnosia represents an impairment in the perception and use of spatial-dependent information, resulting from brain pathology. It refers to an acquired inability to recognize and integrate spatial information. There is no existing primary sensory defect to explain it (Ardila and Rosselli, 1992). Spatial agnosia includes impairment in the recognition of line orientation, defects in depth perception, impairment in handling spatial information, and deficits in spatial memory (Hécaen and Albert, 1978; De Renzi, 1982).

Different types of spatial agnosia have been distinguished. Holmes (1918) separates different categories of spatial agnosia: defects in object localization, topographic amnesia, inability to count objects, inability to perceive movement, loss of stereoscopic vision, and deficits in eye movements. Critchley (1968) includes the following groups: (1) disorders in spatial perception with regard to three-dimensional perception; (2) disorders in spatial concepts; and (3) disorders in spatial manipulation, which includes disorders in topographical memory, defects in orientation, and unilateral spatial agnosia. Hécaen (1962) proposed: (1) disorders in spatial perception; (2) defects in spatial manipulation, including the loss of topographical concepts, and unilateral spatial agnosia; (3) loss of topographical memory; and (4) Balint's syndrome. De Renzi $(1982,1985)$ presents some modifications to Hécaen's classification: Balint's syndrome is included within visual exploration disorders, and instead of disorders in manipulation of spatial information, the group of disorders in spatial thought is introduced. Table I presents a tentative classification of visuospatial disorders.

It is interesting to note that all these disorders appear (mainly or exclusively) in cases of right hemisphere pathology. In general, the right hemisphere seems to be specialized in spatial cognition. Seemingly, language and ideomotor praxic abilities developed in a left brain area, and the right brain is involved in spatial cognition (LeDoux, 1984).

Supposedly, similar spatial cognition disturbances might be found in every species member, regardless of the cultural background and the environmental demands. However, not only commonality but also differences would be expected to be found. If the degree (not the direction) of brain lateralization of language depends on literacy, and in general, on the verbal training history 
TABLE I. Classification of spatial agnosias (according to Ardila and Rosselli, 1992).

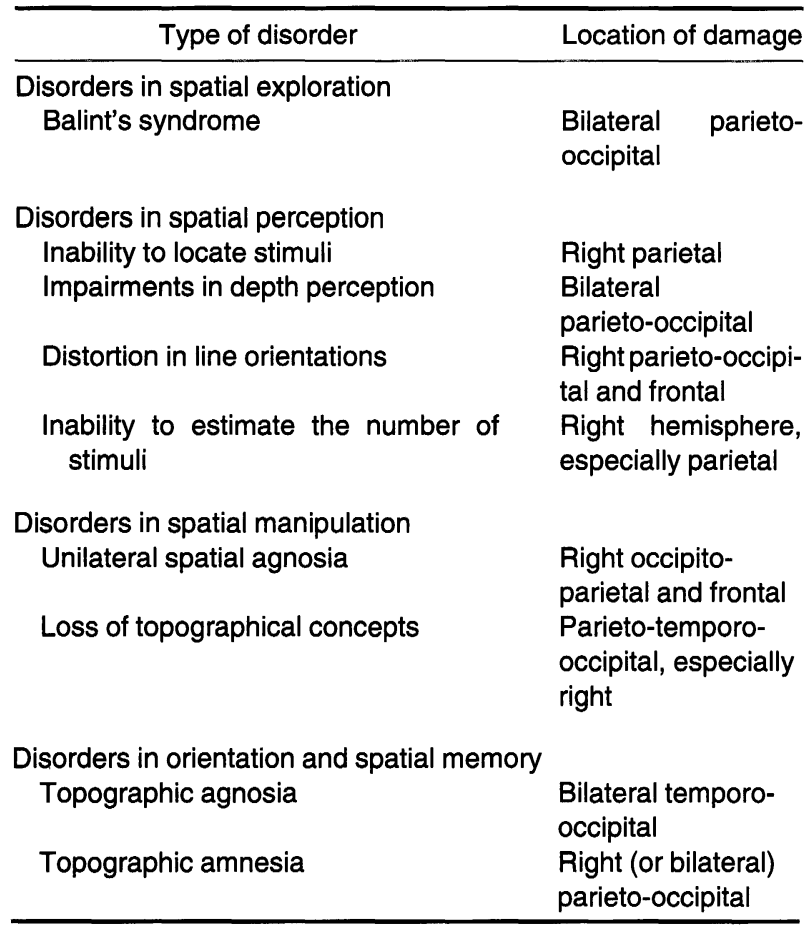

(Lecours et al., 1987, 1988; Matute, 1988), it would seem reasonable to suppose that the degree of lateralization of spatial cognition would also depend on the spatial abilities training history. At least some spatial disturbances (e.g. hemi-spatial neglect) have been reported to be more frequently observed associated with left hemisphere pathology, in individuals with a history of low verbal training (but normal, and eventually superior training in spatial abilities) (Rosselli et al., 1985).

If, despite existing basic characteristics in brain organization, language disturbances (oral and written) are associated with language idiosyncracies (i.e. aphasia is not completely equivalent in Chinese and Spanish; alexia can be different in English and Japanese, etc.; Sasanuma and Fujimura, 1971; Yamadori, 1975; Yu-Huan et al., 1990), spatial cognition disturbances may also depend on spatial ability training histories. This, of course, has to be demonstrated, and it can be demonstrated only by analyzing spatial disturbances in individuals with different cultural backgrounds and different spatial demands.

Nonetheless, contemporary city human's spatial abilities are not necessarily inferior to prehistorical human's or Amazonian Indians' spatial abilities. Spatial abilities may have evolved with the new living and cultural conditions (in a similar way as spoken language evolved and extended with the development of new cultural conditions, e.g. through written language). Spatial abilities are required in many contemporary conceptual, and historically recent, skills. The author of this paper had the opportunity to study a chemistry university professor who suffered a small right parietal infarction. Although she did not have any evident spatial difficulty in her everyday activities, she could not continue teaching chemistry, because she was "unable to have a spatial representation of molecules and all the time got confused". Mathematics (Luria, 1977; Ardila and Rosselli, 1990), painting, playing chess (Chabris and Hamilton, 1992), reading and writing (Ardila and Rosselli, 1993; Benson and Ardila, in press), mechanics (Benton, 1989), and even music (Henson, 1985), all represent, at least partially, spatially based skills. Mathematics, painting, playing chess, reading and writing, mechanics and music abilities can be impaired in cases of right hemisphere damage; in an Eskimo or Amazonian Indian damage of those same areas could impair their ability to move around the snow or the jungle.

\section{CONCLUSIONS}

Homo sapiens have led a nomadic way of life during the majority of their history. A sedentary way of life appeared only with domestication of animals and development of agriculture, some 10000 years ago. A nomadic way of life is strongly associated with high spatial ability demands.

Disorders in spatial cognition represent a particularly complex and insufficiently understood array of impairments. Spatial knowledge has been strongly associated with right hemisphere activity. Virtually all defects in spatial perception and orientation are found exclusively or predominantly in cases of right hemisphere damage.

It might be supposed that right hemisphere specialization for spatial abilities can be correlated with language acquisition and evolution. Furthermore, it has been suggested that right hemisphere specialization in spatial skills, as well as left hemisphere specialization in verbal and praxic abilities, is found to be correlated with literacy, that is, with language complexity. Spoken language evolved with the appearance of new cultural conditions, and it might be supposed that spatial abilities also evolved with the appearance of new cultural and environmental conditions.

It might be proposed that early hominids and prehistoric humans presented a more bilateral representation of spatial abilities. Visuospatial disorders might have been expected in cases of right and left hemisphere pathology. Not only language development and complexity, but also the development of new spatially based abilities may have increased the right hemisphere specialization for handling information with a spatial content, as well as the left specialization for linguistic abilities. 


\section{REFERENCES}

Ardila A (1980) Psicologia de la Percepcion [Psychology of Perception]. Editorial Trillas, Mexico.

Ardila A and Ostrosky F (1984) Some final remarks. In: The Right Hemisphere: Neurology and Neuropsychology (Eds A Ardila and F Ostrosky-Solis), pp. 265-273. Gordon and Breach, London.

Ardila A and Rosselli M(1990) Acalculias. Behavioural Neurology, 3, 39-48.

Ardila A and Rosselli M (1992) Neuropsicologia Clinica [Clinical Neuropsychology]. Prensa Creativa, Medellin, Colombia.

Ardila A and Rosselli M(1993) Spatial agraphia. Brain and Cognition, 22, 75-95.

Ardila A, Rosselli M and Rosas P (1989) Neuropsychological assessment in illiterates: Visuospatial and memory abilities. Brain and Cognition, 11, 147-166.

Benson DF and Ardila A (in press) Aphasia: A Clinical Perspective. Oxford University Press, New York.

Benton A (1989) Constructional apraxia. In: Handbook of Clinical Neuropsychology, Vol. 2 (Eds H. Goodglass and AR Damasio), pp. 287-294. Elsevier, Amsterdam,

Berry JW (1971) Ecological and cultural factors in spatial perceptual development. Canadian Journal of Behavioral Sciences, 3, 324-336.

Berry JW (1979) Culture and cognition style. In: Perspectives in Cross-Cultural Psychology (Eds A Mrsella, RG Tharp and TJ Ciborowski), pp. 117-135. Academic Press, New York.

Beveridge WM (1940) Some racial differences in perception. British Journal of Psychology, 30, 57-64.

Brislin RW (1983) Cross-cultural research in psychology. Annual Review of Psychology, 34, 363-400.

Chabris CF and Hamilton SE (1992) Hemispheric specialization for skilled perceptual organization by chessmasters. Neuropsychologia, 30, 47-57.

Childe VG (1936) Man Makes Himself. Pitman, London.

Critchley M (1968) Clinical considerations on parietal lobe, In: Parietal Lobe (Eds M Velasco and F Escobedo). Instituto Mexicano de Neurologia, Mexico.

De Renzi E (1982) Disorders of Space Exploration and Cognition. Wiley, New York.

De Renzi E (1985) Disorder of space exploration. In: Handbook of Clinical Neurology: Clinical Neuropsychology, Vol 45 (Ed. JAM Frederiks), pp. 405-422. Elsevier, Amsterdam.

Gay J and Cole M (1967) The New Mathematics and an Old Culture. Holt, Rinehart \& Winston, New York.

Gladwin T (1970) East is a Big Bird: Navigation and Logic in Puluwatatoll. Harvard University Press, Cambridge, MA.

Hécaen H (1962) Clinical symptomatology in right and left hemisphere lesions. In: Interhemispheric Relations and Cerebral Dominance (Ed. V.B. Mountcastle), pp. 215-243. John Hopkins, Baltimore.

Hécaen H and Albert ML (1978) Human Neuropsychology. Wiley, New York.

Henson RA (1985) Amusia. In: Handbook of Clinical Neurology: Clinical Neuropsychology, Vol. 5 (Ed. JAM Frederiks), pp. 483-490. Elsevier, Amsterdam.

Holmes G (1918) Disturbances of visual orientation. British Journal of Ophthalmology, 2, 449-486.

Hours F (1982) Les Civilisations du Paléolithique. Presses Universitaires de la France, Paris.
Hudson W (1960) Pictorial depth perception in subcultural groups in Africa. Journal of Social Psychology, 52, 193-208.

Hudson W (1962) Cultural problems in pictorial perception. South African Journal of Sciences, 58, 189-195.

Laboratory of Comparative Human Cognition (1983) Culture and cognitive development. In: Handbook of Child Psychology: History, Theory and Methods, Vol. 1 (Ed. P Mussen), pp.342-397. Wiley, New York.

Lecours RL, Mehler J, Parente MA, Caldeira A et al. (1987) Illiteracy and brain damage 1: aphasia testing in culturally contrasted populations (control subjects). Neuropsychologia, 25, 231-245.

Lecours AR, Mehler J, Parente MA et al. (1988) Illiteracy and brain damage III: A contribution to the study of speech and language disorders in illiterates with unilateral brain damage (initial testing). Neuropsychologia, 26, 575-589.

LeDoux JE (1984) Cognitive evolution: clues from brain asymmetry. In: The Right Hemisphere: Neurology and Neuropsychology (Eds A Ardila and F Ostrosky-Solis), pp. 51-60. Gordon and Breach, London.

Lee RB and DeVore I (1968) Man the Hunter. Aldine, Chicago.

Luria AR (1977) Higher Cortical Functions in Man. Basic Books, New York.

Matute E (1988) El aprendizaje de la lectoescritura y la especialización hemisférica para el lenguaje. In: Lenguaje Oral y Escrito (Eds A Ardila and F Ostrosky-Solis), pp. 310-338. Editorial Trillas, Mexico.

Morrow L and Ratcliff G (1988) The neuropsychology of spatial cognition. In: Spatial Cognition: Brain Bases and Development (Eds J Stiles-Davis, M Kritchevsky and U Bellugi), pp. 5-32. Lawrence Erlbaum Associates, Hillsdale.

Myambo K (1972) Shape constancy as influenced by culture, Western education, and age. Journal of Cross-Cultural Psychology, 3, 221-232.

Newcombe F and Ratcliff G (1989) Disorders of visuospatial analysis. In: Handbook of Clinical Neuropsychology, Vol. 2 (Eds H Goodglass and AR Damasio), pp. 333-356. Elsevier, Amsterdam.

Pick AD and Pick HL, (1978) Culture and perception In: Handbook of Perception, Vol. 10: Perceptual Ecology (Eds EC Carterette and MP Friedman), pp. 19-39. Academic Press, New York.

Rosselli M (1986) Conocimiento espacial y sus alteraciones. Acta Neurológica Colombiana, 2, 5-10.

Rosselli M, Rosselli A, Vergara I and Ardila A (1985) The topography of the hemi-inattention syndrome. International Journal of Neuroscience, 20, 153-160.

Sasanuma S and Fujimura O (1971) Kanji versus Kana processing in alexia with transient agraphia. Cortex, 7, 1-18.

Segall MH (1986) Culture and behavior: Psychology in global perspective. Annual Review of Psychology, 37, 523-564.

Vygotsky LS (1962) Thought and Language. Massachusetts Institute of Technology Press, Cambridge, MA.

Washburn SL (1978) The evolution of man. Scientific American, 239(3), 194-207.

Wilson AD (1975) Sociobiology. The Belknap Press of the Harvard University Press, Cambridge, MA.

Yamadori A (1975) Ideogram reading in alexia. Brain, 98, 231-298.

Yu-Huan H, Ying-Guan Q and Gui-Qing Z (1990) Crossed aphasia in Chinese: A clinical survey. Brain and Language, 39, 347-356. 


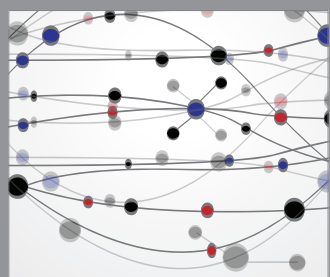

The Scientific World Journal
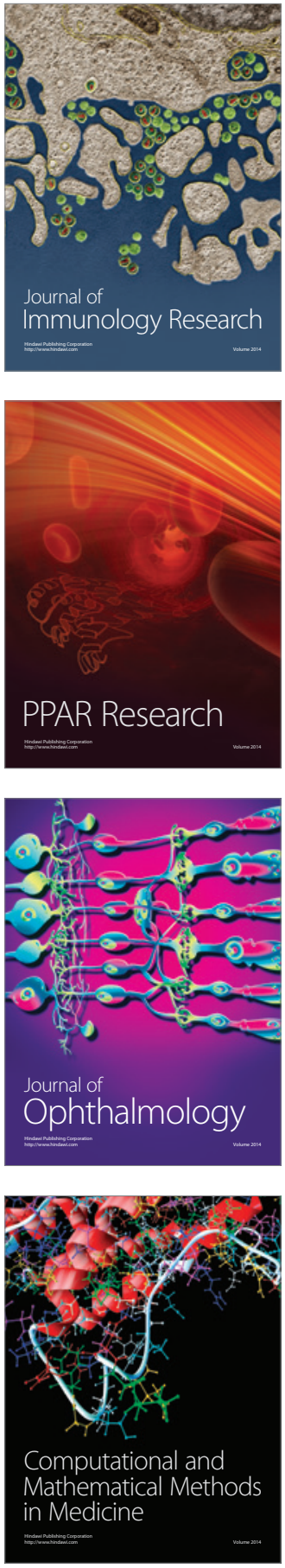

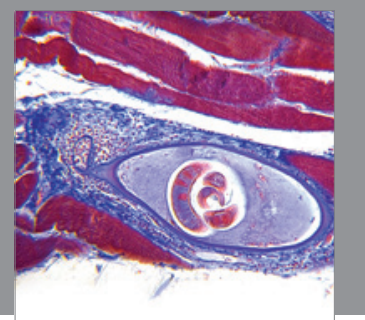

Gastroenterology

Research and Practice
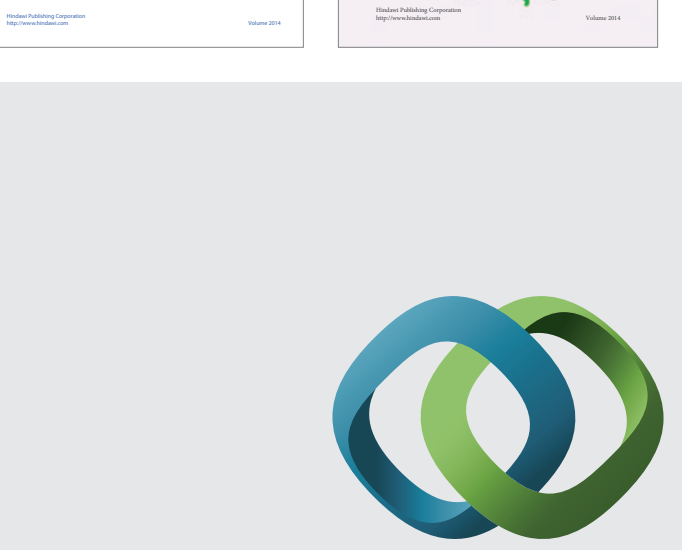

\section{Hindawi}

Submit your manuscripts at

http://www.hindawi.com
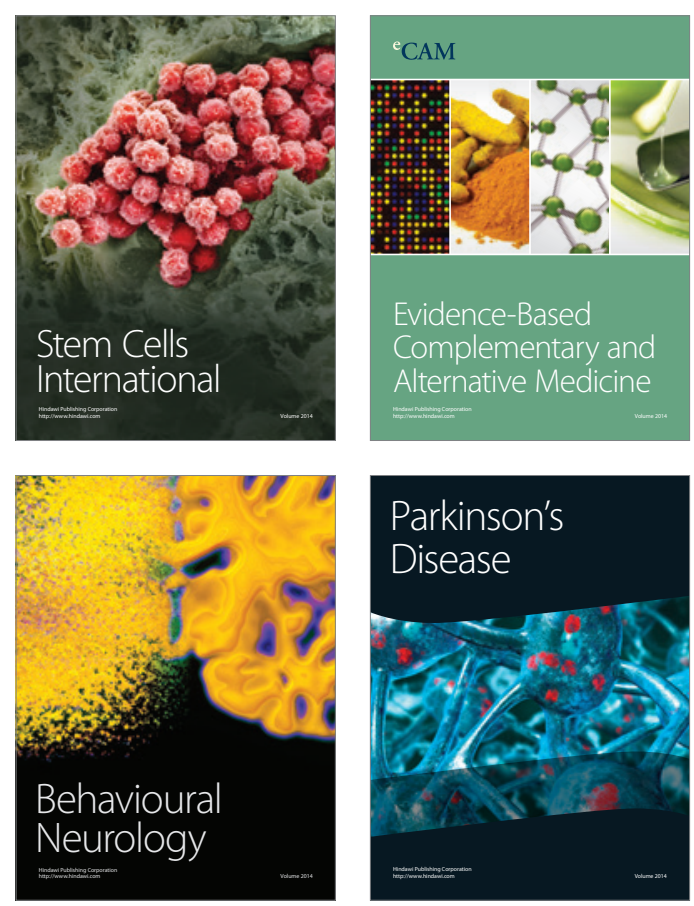

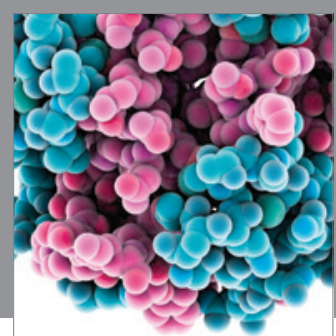

Journal of
Diabetes Research

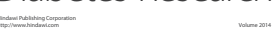

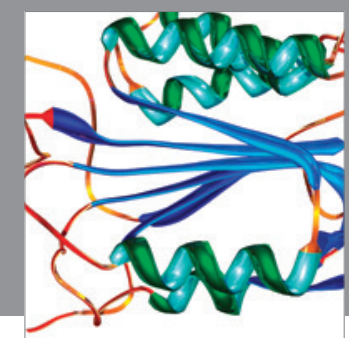

Disease Markers
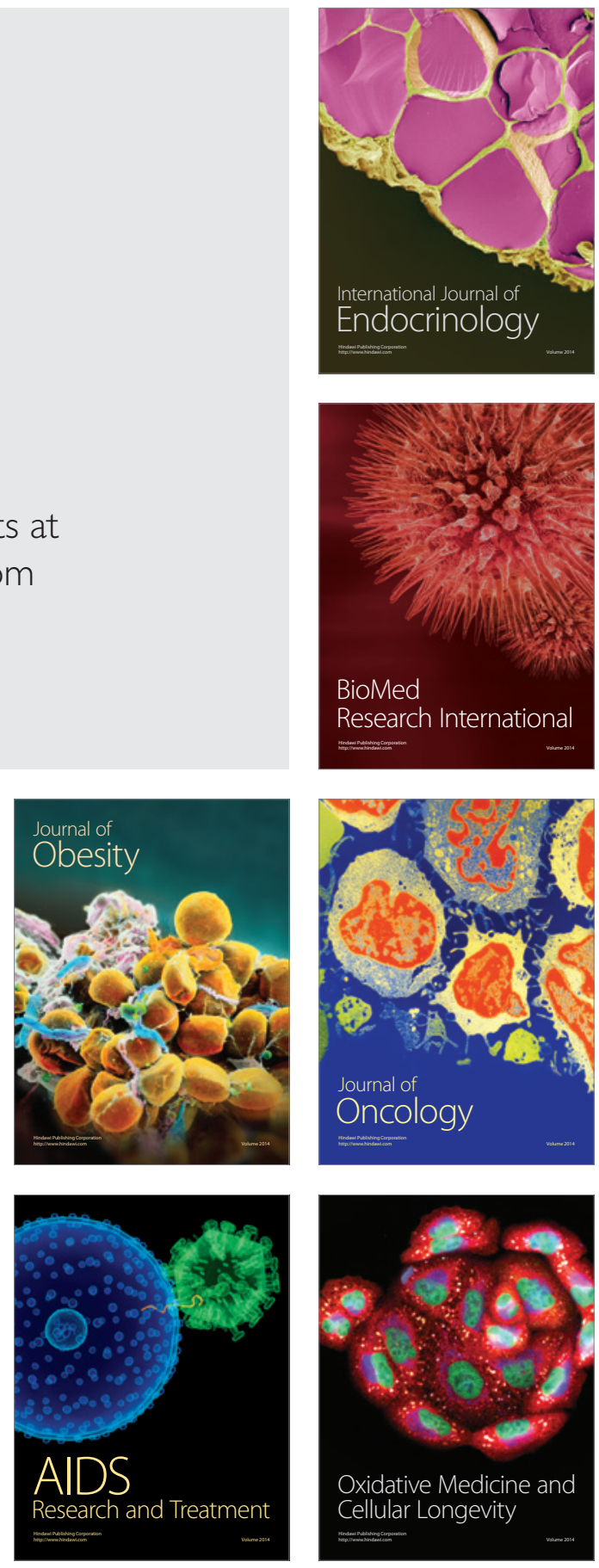